\title{
A Scoping Study of Psychosocial Factors in Women Diagnosed with and/or Treated for Pelvic Organ Prolapse
}

\author{
Kaitlin K. Touza, MS* \\ Kevin L. Rand, $\mathrm{PhD}^{*}$ \\ Janet S. Carpenter, PhD, RN, FAAN $\dagger$ \\ Chen X. Chen, $\mathrm{PhD}, \mathrm{RN} \dagger$ \\ Michael H. Heit, MD, PhD $\$$ \\ *Department of Psychology, School of Science, Indiana University-Purdue University \\ Indianapolis \\ $\dagger$ Science of Nursing Care Department, School of Nursing, Indiana University \\ $\$$ Department of Obstetrics and Gynecology, School of Medicine, Indiana University
}

Corresponding author: Kaitlin K. Touza, MS, Department of Psychology, School of Science, Indiana University-Purdue University Indianapolis, 402 N. Blackford St. LD124, Indianapolis, $\mathrm{IN}, 46204$ 636-352-3177

317-274-6756 (fax)

This is the author's manuscript of the article published in final edited form as:

Touza, K., Rand, K., Carpenter, J., Chen, C., \& Heit, M. (2018). A Scoping Study of Psychosocial Factors in Women Diagnosed With and/or Treated for Pelvic Organ Prolapse. Female Pelvic Medicine \& Reconstructive Surgery, Publish Ahead of Print. https://doi.org/10.1097/SPV.0000000000000578 


\begin{abstract}
Objective: Pelvic organ prolapse (POP) is prevalent and can impact women's physical and psychosocial health. To develop interventions that support this population, an understanding of the state of research on psychosocial factors related to POP is essential. We conducted a scoping study focused on the psychosocial experience of women with POP. The purpose of this review was to (1) inventory and describe the current state of knowledge of the psychosocial experience of women with POP, (2) identify gaps in knowledge, and (3) to identify targets for future research. Method: Electronic databases PsycINFO, PubMed, Embase, and Cumulative Index to Nursing and Allied Health Literature (CINAHL) were searched through November 1, 2017. Results: Of 524 titles reviewed, 103 articles met all inclusion criteria. Articles were grouped by the disease period (i.e., pre-diagnosis, diagnosis/pre-intervention, intervention, follow-up, and mixed), and psychosocial factors. Most articles $(n=73)$ focused on women undergoing intervention. Articles focusing on the pre-intervention period was the next largest category $(\mathrm{n}=$ 14). Follow-up after intervention $(n=8)$ and samples of mixed disease periods $(n=7)$ were less common. One article focused on women before diagnosis. Articles focused on quality of life (QOL; $n=79)$, sexual function $(n=51)$, satisfaction $(n=16)$, body image $(n=13)$, psychological distress $(\mathrm{n}=4)$, and knowledge $(\mathrm{n}=3)$. Conclusion: Research on the psychosocial experience of POP has largely focused on changes in QOL and sexual function. Future research should target: emotional experience of women with POP; relationships among QOL, psychological distress, body image, and sexual function; and psychosocial factors related to treatment outcomes.
\end{abstract}

Keywords: Pelvic organ prolapse, quality of life, psychosocial, distress, sexual function 


\section{$\underline{\text { Introduction }}$}

Pelvic organ prolapse (POP) is symptomatic in $2.9 \%$ of US women $[1,2]$ and is surgically corrected in $12.6 \%$ of cases $[3,4]$. POP occurs when pelvic muscles and tissues weaken or fail, resulting in the descent of pelvic organs (i.e., bladder, uterus, and small and large bowel), sometimes to the point that the vagina and uterus are visible outside of the body [4-6]. The development of POP is multifactorial and is most consistently related to vaginal parity, higher body mass index (BMI), and increasing age $[4,6]$. Advanced POP is commonly defined as the leading edge of prolapse greater than $1 \mathrm{~cm}$ beyond hymen, while minimal POP reflects the leading edge at or above $1 \mathrm{~cm}$ beyond the hymen $[4,7]$. With more advanced anatomical POP, the descending pelvic organs can displace the urethra and/or rectum leading to difficulty with urinary and/or fecal elimination or incontinence $[4,8]$. POP can limit or impair physical functioning and sexual functioning, two important components of quality of life (QOL) [4, 9, $10]$.

It is unclear how much is known about women's psychosocial experience in relation to POP. For the purpose of this review, psychosocial experience is defined as factors related to psychological and social function and well-being. These factors include psychological distress (i.e., depressive symptoms, anxiety, and stress), self-perceived body image, regret and satisfaction related to POP treatment, and sexual function. For the purpose of this review, QOL is also included in the umbrella term psychosocial experience. QOL is a broad construct, incorporating many factors related to the psychosocial experience of women with POP. QOL encompasses both general health-related QOL (i.e., physical, mental, emotional, and social functioning) [11] and POP-specific QOL (i.e., the presence, intensity, and interference of POPrelated symptoms) $[12,13]$. 
To the best of our knowledge, there is no published review focused on the psychosocial experience related to the diagnosis and treatment of POP. In other illness states, the psychosocial experience of disease can profoundly affect treatment outcomes [14]. For example, pre-operative depression predicts morbidity following coronary bypass surgery[15], pre-operative psychological distress and catastrophizing predict pain following total knee arthroplasty[16], and psychological distress and dysfunction are risk factors for poor outcomes following back surgery [17]. The psychosocial experience may be particularly important in predicting pain and other outcomes in elective surgery [14]. Thus, it seems reasonable to explore women's psychosocial experiences related to the diagnosis and treatment of POP.

We conducted a scoping study focused on the psychosocial experience of women diagnosed with and/or treated for POP. Our aims were consistent with the widely accepted purpose of a scoping study. A scoping study aims to summarize the state of research on a topic that may have been previously neglected, or has not yet been reviewed, and to identify gaps in knowledge and targets for future research [18]. It differs from a meta-analysis or systematic review in that it aims to assess the scope of research on a particular subject without focusing on a specific research question [18]. A scoping study incorporates a wide range of study designs and addresses questions beyond intervention efficacy, as is done in a meta-analysis or systematic review, and therefore provides a more comprehensive summary of the totality of available literature on a topic.

\section{Materials and Methods}

To complete the scoping study, we followed Arksey and O’Malley's [18, 19] framework and guidelines. Their 5 stages for investigating a review topic are: (1) identifying the research 
problem, (2) identifying relevant studies, (3) study selection, (4) charting the data; and (5) collating, summarizing, and reporting the results.

\section{Identifying the research problem}

With this research, we aimed to answer the following questions: (1) what is the current state of knowledge of the psychosocial experience of women with POP; (2) where are there gaps in knowledge; and (3) what future research is needed? We did not comment on the quality of research included and this was consistent with the primary purpose and philosophy of scoping studies, which is to map out the current literature on a topic. Adopting a less conservative approach to study inclusion for a scoping study ensures that studies of varying design and methodology are included.

\section{Identifying relevant studies}

The electronic databases PsycINFO, PubMed, Embase, and the Cumulative Index to Nursing and Allied Health Literature (CINAHL) were searched through November 1, 2017. No restrictions on publication date were applied. Search terms were based on the medical definition of POP. Psychosocial search terms were adapted from a review paper examining the psychosocial experience of women with endometriosis, which shares many salient features with POP (i.e., (1) the disease affects the sexual organs in women, (2) it is a painful and hidden condition, (3) diagnosis is difficult without physical examination, (4) treatment often requires surgery, (5) it impacts sexual function, and (6) it is associated with changes in body image, psychological well-being, and QOL [20].

The following search terms were used for each database: (pelvic organ prolapse OR pelvic prolapse OR organ prolapse OR uterine prolapse OR apical prolapse OR cystocele OR 
rectocele OR sigmoidocele OR enterocele) AND (coping OR distress OR psychological distress OR emotion OR psychosocial OR psychosocial well-being OR psychological well-being OR subjective well-being OR well-being OR affect OR identity OR sexual function OR stigma OR expectations OR quality of life OR QOL OR anxiety OR depression OR depressive OR mental health OR body image OR self-image OR personality OR social support OR social well-being OR health literacy). In this study, sexual function refers to several domains of the sexual experience (i.e., arousal, orgasm, satisfaction, lubrication, dyspareunia, and perceptions of partner satisfaction and experience).

\section{Study Selection}

Articles were screened based on the following inclusion and exclusion criteria. Studies needed to be: (1) published in a peer-reviewed journal, (2) available in English, and (3) focused on the following topics: (a) psychosocial variables in women diagnosed with POP, (b) healthrelated or symptom-related QOL associated with POP or intervention for POP, (c) the psychosocial experience before and/or after intervention for POP, (d) psychosocial outcomes associated with different surgical or non-surgical interventions, or (d) patient-reported symptoms or functional outcome associated with POP or intervention for POP. Because we sought to be inclusive of all relevant studies, we did not exclude studies by methodology. Quantitative, qualitative, pilot, and exploratory studies were all considered for inclusion. Studies were excluded if: (1) they focused solely on validating survey measures or (2) their primary purpose was on the comparative efficacy of medical interventions without a focus on the psychosocial experience.

Charting the data 
For each included article, the following information was extracted: (1) author and year of publication; (2) research aim; (3) reported psychosocial variables (4) standardized measures of psychosocial variables, if reported; (5) number of participants included in the analyses; (6) mean age and standard deviation of the sample, if reported; (7) authors' conclusions; and (8) period of disease the sample was in. The period of disease was categorized as (1) pre-diagnosis, (2) diagnosis/pre-intervention, (3) intervention, (4) follow-up, and (5) mixed. For articles that focused on patients in the intervention and follow-up stages, the number of time points and follow-up schedule was also noted.

\section{$\underline{\text { Results }}$}

A total of 524 unique articles were reviewed. One hundred and three articles met all inclusion criteria and are summarized in this review (Figure 1). The results are organized according to disease period and supplemented by tables. Table 1 summarizes measurement tools used to assess the psychosocial experience. Table 2 provides an overall summary of the studies and findings. Table 3 describes the psychosocial experience by disease.

The articles were categorized into the following disease periods: (1) pre-diagnosis $(n=1$, $1.0 \%) ;(2)$ diagnosis/pre-intervention $(n=14,13.6 \%) ;(3)$ intervention $(n=73,70.9 \%)$; (4) follow-up $(n=8,7.8 \%)$; and $(5)$ mixed $(n=7,6.8 \%)$. Eight categories of psychosocial variables were found in the included studies: (1) POP-specific QOL ( $\mathrm{n}=77,74.8 \%)$; (2) sexual function ( $\mathrm{n}$ $=51,49.5 \%)$; (3) satisfaction $(\mathrm{n}=16,15.5 \%)$; (4) general health related QOL $(\mathrm{n}=12,11.7 \%)$;

(5) body image $(n=13,12.6 \%)$; (6) knowledge of POP $(n=6,5.8 \%)$; and (7) psychological distress $(n=4,3.9 \%)$. Not all variables related to psychosocial experience were reported in each disease period (Table 3$)$. 


\section{Pre-diagnosis}

One study focused on prevalence of POP-related symptoms, awareness of several pelvic floor disorders in family members, and understanding of factors that contribute to these disorders in young and adolescent women not diagnosed with POP [21]. Few women reported symptoms, were aware of POP in family members, or knew about factors that contribute to POP [21].

\section{Diagnosis/pre-treatment}

\section{Quality of life}

There was no difference in overall POP-specific QOL by prolapse type [22]. Women with more severe anatomical POP reported more POP-related symptoms (i.e., worse POPspecific QOL) [12]. Patient treatment goals also differed by severity of anatomical POP, though the goals reported centered around resolution of symptoms and improvement of POP-specific QOL, regardless of POP stage [23]. Women with POP had worse general health related QOL in the physical domain and worse POP-specific QOL than healthy women, but there was no difference between groups in the mental domain of general health related QOL [24]. Women awaiting surgery for POP reported similar deficits in general health related QOL as women awaiting hip or knee replacement [25]. The wait time for POP surgery was more than twice that for hip or knee replacement [25]. Pelvic floor muscle function was not associated with POPspecific QOL [26].

\section{Sexual function}

Sexual function was not related to minimal anatomical POP [27]. However, women with more severe anatomical POP reported worse sexual function than healthy women [28]. There was no difference in sexual function by prolapse type [22]. 
Knowledge of $P O P$

Women had poor understanding of POP and its treatment $[29,30]$, even with high health literacy [29]. Women reported poor disease understanding and knowledge of POP as barriers to seeking treatment $[31,32]$. Women reported that they are not being educated about POP, risk factors, or treatment options by their health care providers [31], and that feelings of guilt and blaming oneself also kept them from seeking treatment [32].

\section{Body image}

Women with POP reported worse self-perceived body image [24] and having more severe POP-related symptoms was associated with worse body image [33]. Poorer body image correlated with poorer scores on the mental domain of QOL, regardless of presence of POP [24].

\section{Psychological distress}

Women who reported more depressive symptoms reported more severe POP-related symptoms and worse general health related QOL, regardless of the severity of anatomical POP [34].

Intervention

Studies in the intervention period focused on changes in psychosocial experience before and after intervention.

POP-specific quality of life

All studies found that POP-specific QOL improved after reconstructive surgery [35-65]. There was no difference in improvement in POP-specific QOL following surgery by age group [66]. Women who had undergone obliterative surgery also reported improved POP-specific QOL 
[46, 67-70]. Use of a pessary was also associated with improved POP-specific QOL [45, 71-73]. Biofeedback paired with pelvic floor muscle training (PFMT) improved POP-specific QOL [74]. Follow up periods for outcome measurement varied widely, from six months to five years.

One study examined pre-intervention scores on two measures of POP-specific QOL as predictors of improvement in POP-specific QOL following intervention [60]. Women with worse POP-specific QOL at baseline were more likely to report improvement at follow-up [60].

\section{General health related quality of life}

General health related QOL also improved following reconstructive surgery [61, 66, 7579]. Women who underwent obliterative surgery also reported improvement in general health related QOL [80]. Pessary use was also associated with improved general health related QOL [78]. Follow-up ranged from three to 18 months.

\section{Satisfaction}

Most women reported being satisfied after reconstructive surgery [43, 46, 47, 49, 81-83]. Satisfaction following reconstructive surgery was associated with patient preparedness for surgery [84], patient treatment-related goal achievement [85], age at surgery and follow-up [83], menopausal status [83], BMI [83], and presence of POP symptoms [83]. Women who underwent obliterative surgery also reported being satisfied $[46,67,70,80]$.

\section{Sexual function}

Outcomes for sexual function following reconstructive surgery varied. Most studies reported improvement in sexual function following reconstructive surgery $[35,41,42,54,57,58$, $61,63,65,79,86-92]$. Sexual function improved similarly with abdominal and transvaginal 
surgery [65]. Women who underwent a hysterectomy [81, 91, 93] or a trachelectomy [82] concurrent with surgical repair of POP also reported improvement in sexual function. Male partner sexual function improved following surgery in one study [89], but no change was reported in another [86]. Follow-up ranged from three months to three years following surgery.

Several studies reported no change in sexual function following reconstructive surgery $[36,37,39,66,86,94-99]$. Other studies reported declines in sexual function following reconstructive surgery $[51,62,100]$. Postmenopausal women reported worse sexual function after surgery than premenopausal women [101]. Follow-up ranged from six months to three years after surgery.

The impact of non-surgical interventions on sexual function also varied. There was no difference in sexual function between women who had undergone PFMT and those who did not, however, more women in the PFMT group reported improved sexual function [102]. Two studies examining pessary use reported no change in sexual function [103, 104], while another study reported that sexual function improved with pessary use [78].

Several factors were identified that may influence sexual function outcomes. Follow-up period may impact outcomes, as sexual function declined immediately following surgery but improved over time, in one study [105]. Site of reconstructive surgery may also impact sexual function outcomes, as one study found that women who had anterior or posterior repair alone, reported improvement in sexual function, while women who had both anterior and posterior repair reported no change [106]. In one study, reduced coital incontinence and better overall health predicted improvement in sexual function following surgery, while psychological distress predicted decline [100]. However, another study found that sexual function was driven by 
behavioral-emotive (i.e., desire) and partner related (i.e., ability to maintain an erection) domains rather than factors in the physical domain (i.e., pain) [107].

\section{Body image}

Reconstructive surgery $[45,61,78]$, use of a pessary $[45,73]$, and obliterative surgery $[70,80]$ were associated with improvement in self-perceived body image.

\section{Psychological distress}

Women with POP reported more depressive symptoms than women without POP, and depressive symptoms improved six months after surgery [108]. Rural Nepalese women who had worse general health related QOL, also had worse depression scores, and both depression and general health related QOL improved three months following surgery [77].

\section{Follow-up}

Follow-up articles examined psychosocial factors after intervention but did not compare these factors to baseline levels.

Women with POP were compared to women without POP after having previously undergone surgical removal of the cervix; women without POP reported greater satisfaction, better general health related QOL, and better sexual function [109].

Women who had previously undergone transvaginal reconstructive surgery reported good POP-specific QOL [110]. Women who had previously undergone obliterative surgery reported deficits in sexual function [111] and body image [111, 112]. However, most reported low regret, improvement in POP-specific QOL, and were satisfied [111-113]. Women who reported better POP-specific QOL after surgery were more likely to be satisfied [114]. 
Women with recurrent POP reported similar barriers to seeking treatment as treatment naïve women, including beliefs about aging, availability of treatment, trivializing the importance of symptoms, and the high workload of doctors [115].

\section{Mixed}

This category includes studies that focused on women in various disease periods.

Women who reported more POP-related symptoms, had worse general health related QOL [116]. Women in the $6^{\text {th }}$ and $7^{\text {th }}$ decade of life were more bothered by POP-related symptoms than women who were younger or older with the same stage of prolapse [117].

Women with POP reported worse sexual function in both behavioral-emotive and physical domains, compared to women without POP [118]. Deficits in sexual function were associated with worse body image and more severe POP-related symptoms, regardless of POP stage [119]. Women with POP that had not been surgically corrected reported worse genital body image than women without POP, but women with surgically corrected POP did not differ from either group [120]. Worse genital body image was uniquely associated with worse sexual function in all groups, when general body image was controlled for [120].

Women reported shame, anxiety, frustration, hopelessness, and acceptance related to POP [9]. Deficits in self-image, social activity (i.e., being more housebound), hygiene, and discomfort were reported [9].

Approximately one-third of women with symptomatic prolapse reported depressive symptoms [121]. Depressive symptoms were related to urinary tract symptoms and bowel dysfunction but not prolapse stage [121]. 


\section{$\underline{\text { Discussion }}$}

In this scoping study, we mapped out literature on the psychosocial experience of women diagnosed with and/or treated for pelvic organ prolapse (POP). The first major conclusion of this review is that there is considerable variability in what outcomes have been measured and the length of follow-up after intervention. QOL and sexual function were the most consistently reported variables, whereas variables such as psychological distress and knowledge of POP were relatively neglected. There was a wide range in the length of follow-up reported, from a few months to several years. One study found that sexual function declined in the first three months after surgery, and then improved over time, such that it had reached statistically significant improvement at two years after surgery [105]. Researchers and clinicians should work together to develop a toolkit of suggested measures and assessment timeframes.

The second major conclusion of this review is that because the impact of psychosocial factors on treatment outcomes, such as recovery, was unexplored, more research is needed to examine psychosocial predictors of treatment outcomes in POP. Psychological distress predicts outcomes in other surgeries [14-17], and the impact of psychological distress on outcomes in POP treatment should be examined. For example, in healthy women, psychological distress is known to impact sexual function [122] but the relationship between psychological distress and sexual function outcomes has not been explored in women with POP. Also, women with worse depressive symptoms reported worse POP-related symptoms, regardless of the severity of anatomical POP [34]. How psychological well-being impacts the subjective experience of POP has not been adequately explored. Further, factors, such as expectancy [123], that have been shown to predict treatment outcomes in other diseases, were not explored in women with POP. In a sample with mixed pelvic floor disorders, trait optimism predicted subjective report of 
pelvic floor symptoms, such that those with greater trait positive expectancy reported less symptoms and less distress related to symptoms[124]. The ability of positive expectancies to predict treatment outcomes in POP should be explored.

The third major conclusion is that significant gaps exist in the current literature that justify future research. Such gaps preclude our understanding of the POP psychosocial experience and interfere with the ability to provide evidence-based care to improve psychosocial outcomes. The five main areas where future research is needed are described below.

First, future research should determine the relationship between POP and psychological distress, body image, and sexual function. Women reported concerning deficits in body image, sexual function, and psychological well-being. Future research should clarify how POP impacts these factors and vice versa. As studies found that body image and depression were associated with severity of self-rated symptoms (i.e., worse POP-specific QOL), regardless of severity of anatomical POP $[34,119]$, it is important to determine how deficits in psychological well-being impact the subjective experience of POP.

Second, future research should clarify the impact of POP treatment on sexual function. Sexual function outcomes varied widely after both surgical and non-surgical treatment for POP. Studies reported no change, decline, or improvement following treatment. As POP is consistently associated with deficits in sexual function, and improved sexual function is a commonly measured outcome variable and indicator of treatment success, it is important to establish factors that predict improvement. Factors that were identified as being associated with sexual function outcomes should be expanded upon and confirmed. These factors included the length of followup, coital incontinence, general health status, and type of surgery. 
Third, future research should determine factors that impact or predict treatment outcomes. Factors such as psychological distress [125], expectancy [122], and goal-related traits (i.e., self-efficacy and optimism) $[126,127]$ have been established as predictors of surgical and treatment outcomes (i.e., recovery) in other illnesses. These factors should be examined in women undergoing treatment for POP. Establishing the impact of pre-intervention psychological factors on surgical outcomes may help physicians to optimize treatments and recovery for patients with POP.

Fourth, future research should aim to improve knowledge of POP in health care consumers. Knowledge related to POP was poor [29, 30]. Poor knowledge of POP and understanding of treatment was reported as a barrier to treatment in several studies [31, 32]. Poor understanding of POP and its treatment was reported even by women who had previously undergone treatment for POP [115]. Research focusing on improving knowledge, recognizing and reducing barriers to treatment, and improving treatment seeking in women with symptomatic POP is warranted.

Fifth and finally, future research should establish the influence of length of follow-up on treatment outcomes. The follow-up periods for treatment outcomes varied widely across studies, with some studies reporting outcomes several months after surgery and other reporting outcomes after several years. The impact of length of follow-up on outcomes, such as recovery, should be established. Further, identification of factors that predict the trajectory of outcomes over time (i.e., differential recovery trajectories) may help physicians to counsel patients about surgery and expected outcomes over time.

Our scoping study findings should be interpreted in light of some limitations. First, as this was a scoping study, the sections describing research related to POP and sexual function were 
intentionally broad out of necessity, to give our study group the latitude to be inclusive in other subject areas. A separate review of the psychosocial factors facing women with sexual dysfunction and POP might also be beneficial for the readership. Second, we did not assess the quality of included research, which was consistent with how scoping studies are typically conducted. Additional implications for future research may be identified once the quality of the research is assessed. For example, areas of existing but methodologically poor research may suggest a need for additional research, though many areas in need of research have already been identified in this review. Third, relevant research may have unintentionally been omitted from this review based on inclusion/exclusion criteria, search terms, and/or databases searched. Hand searching of relevant journals was not performed, such that studies that were not available in online databases were not included. This may have resulted in an omission of relevant research. Additionally, some relevant research may have been omitted due to the specificity of the search terms used, as our goal was to be specific to POP and not all pelvic floor disorders. We chose to use specific search terms in order for our methods to be replicable and with the expectation that missing results would be random and the review comprehensive. Fourth, a single reviewer coded the articles in this review. Therefore, the included articles may be subject to selection bias as inclusion and exclusion decisions reflect the opinion of one reviewer. However, this study is strengthened by the significant involvement and authorship of M.H.H.; M.H.H. is a fellowship trained surgeon in the field of female pelvic medicine and reconstructive surgery, who has a $\mathrm{PhD}$ in epidemiology and clinical research and has provided expert consultation from development to completion of this project.

\section{Conclusion}


Research focusing on the psychosocial experience of women with POP is relatively new, with most studies focusing on sexual function and QOL in these women. Future research should focus on factors that predict treatment outcomes, the relationship between POP and psychological distress, knowledge of POP and barriers to treatment, and how the length of the follow-up impacts measured outcomes. Developing a deeper understanding of the psychosocial experience of having this disorder may help clinicians to improve care for their patients and may help consumers better advocate for themselves when seeking healthcare. 


\section{References}

1. Nygaard, I., et al., Prevalence of symptomatic pelvic floor disorders in US women. Jama, 2008. 300(11): p. 1311-6.

2. $\quad$ Wu, J.M., et al., Prevalence and trends of symptomatic pelvic floor disorders in U.S. women. Obstet Gynecol, 2014. 123(1): p. 141-8.

3. Hendrix, S.L., et al., Pelvic organ prolapse in the Women's Health Initiative: gravity and gravidity. Am J Obstet Gynecol, 2002. 186(6): p. 1160-6.

4. Jelovsek, J.E., C. Maher, and M.D. Barber, Pelvic organ prolapse. Lancet, 2007. 369(9566): p. 1027-38.

5. Yousuf, A., et al., The length of anterior vaginal wall exposed to external pressure on maximal straining MRI: relationship to urogenital hiatus diameter, and apical and bladder location. Int Urogynecol J, 2014. 25(10): p. 1349-56.

6. Low, L.K. and J.A. Tumbarello, Falling out: authoritative knowledge and women's experiences with pelvic organ prolapse. J Midwifery Womens Health, 2012. 57(5): p. 489-94.

7. Bump, R.C., et al., The standardization of terminology of female pelvic organ prolapse and pelvic floor dysfunction. Am J Obstet Gynecol, 1996. 175(1): p. 107.

8. Barber, M.D., M.D. Walters, and R.C. Bump, Short forms of two conditionspecific quality-of-life questionnaires for women with pelvic floor disorders (PFDI-20 and PFIQ-7). American Journal of Obstetrics \& Gynecology, 2005. 193(1): p. 103-113.

9. $\quad$ Roets, L., The experience of women with genital prolapse. Curationis, 2007. 30(3): p. 7-14.

10. Şahin, E. and G. Vural, Assessment of the Quality of Life in Women with a Diagnosis of Urogenital Prolapse. International Journal of Caring Sciences, 2015. 8(2): p. 404-411.

11. Ware, J.E., Jr. and C.D. Sherbourne, The MOS 36-item short-form health survey (SF-36). I. Conceptual framework and item selection. Med Care, 1992. 30(6): p. 473-83.

12. Digesu, G.A., et al., The relationship of vaginal prolapse severity to symptoms and quality of life. BJOG: An International Journal of Obstetrics \& Gynaecology, 2005. 112(7): p. 971-976.

13. Digesu, G.A., et al., P-QOL: a validated questionnaire to assess the symptoms and quality of life of women with urogenital prolapse. Int Urogynecol J Pelvic Floor Dysfunct, 2005. 16(3): p. 176-81; discussion 181.

14. Bruns, D. and J.M. Disorbio, Assessment of biopsychosocial risk factors for medical treatment: a collaborative approach. J Clin Psychol Med Settings, 2009. 16(2): p. 127-47.

15. Burg, M.M., et al., Presurgical depression predicts medical morbidity 6 months after coronary artery bypass graft surgery. Psychosom Med, 2003. 65(1): p. 1118.

16. Roth, M.L., et al., Demographic and psychosocial predictors of acute perioperative pain for total knee arthroplasty. Pain Res Manag, 2007. 12(3): p. 185-94. 
17. Block, A.R., Y.S. Ben-Porath, and R.J. Marek, Psychological risk factors for poor outcome of spine surgery and spinal cord stimulator implant: a review of the literature and their assessment with the MMPI-2-RF. Clin Neuropsychol, 2013. 27(1): p. 81-107.

18. Arksey, H. and L. O'Malley, Scoping Studies: Towards a Methodological Framework. International Journal of Social Research Methodology: Theory \& Practice, 2005. 8(1): p. 19-32.

19. Levac, D., H. Colquhoun, and K.K. O'Brien, Scoping studies: advancing the methodology. Implementation Science : IS, 2010. 5: p. 69-69.

20. Melis, I., et al., Sexual function in women with deep endometriosis: Correlation with quality of life, intensity of pain, depression, anxiety, and body image. International Journal of Sexual Health, 2015. 27(2): p. 175-185.

21. Parden, A.M., et al., Prevalence, Awareness, and Understanding of Pelvic Floor Disorders in Adolescent and Young Women. Female Pelvic Med Reconstr Surg, 2016. 22(5): p. 346-54.

22. Ozengin, N., et al., The effect of pelvic organ prolapse type on sexual function, muscle strength, and pelvic floor symptoms in women: A retrospective study. Turk J Obstet Gynecol, 2017. 14(2): p. 121-127.

23. Adams, S.R., et al., Do patient goals vary with stage of prolapse? American Journal of Obstetrics \& Gynecology, 2011. 205(5): p. 502.e1-6.

24. Jelovsek, J.E. and M.D. Barber, Women seeking treatment for advanced pelvic organ prolapse have decreased body image and quality of life. American Journal of Obstetrics \& Gynecology, 2006. 194(5): p. 1455-1461.

25. Leong, Y., et al., A Comparison of Health-Related Quality of Life of Women Awaiting Pelvic Organ Prolapse Surgery versus Hip or Knee Replacement. J Obstet Gynaecol Can, 2017. 39(5): p. 341-346.

26. Borello-France, D.F., et al., Pelvic-floor muscle function in women with pelvic organ prolapse. Physical Therapy, 2007. 87(4): p. 399-407.

27. Karabulut, A., et al., Evaluation of confounding factors interfering with sexual function in women with minimal pelvic organ prolapse. Int J Gynaecol Obstet, 2014. 127(1): p. 73-6.

28. Tok, E.C., et al., The effect of pelvic organ prolapse on sexual function in a general cohort of women. J Sex Med, 2010. 7(12): p. 3957-62.

29. Anger, J.T., et al., Health literacy and disease understanding among aging women with pelvic floor disorders. Female Pelvic Med Reconstr Surg, 2012. 18(6): p. 340-3.

30. Good, M.M., et al., Prolapse-related knowledge and attitudes toward the uterus in women with pelvic organ prolapse symptoms. Am J Obstet Gynecol, 2013. 209(5): p. 481.e1-6.

31. Muller, N., Pelvic organ prolapse: a patient-centred perspective on what women encounter seeking diagnosis and treatment. Australian \& New Zealand Continence Journal, 2010. 16(3): p. 70-80.

32. Pakbaz, M., et al., 'A hidden disorder until the pieces fall into place'--a qualitative study of vaginal prolapse. BMC Women's Health, 2010. 10: p. 18-18. 
33. Lowder, J.L., et al., Body image perceptions in women with pelvic organ prolapse: a qualitative study. American Journal of Obstetrics \& Gynecology, 2011. 204(5): p. 441.e1-5.

34. Pizarro-Berdichevsky, J., et al., Association between pelvic floor disorder symptoms and QoL scores with depressive symptoms among pelvic organ prolapse patients. Australian \& New Zealand Journal of Obstetrics \& Gynaecology, 2016. 56(4): p. 391-397.

35. Bondili, A., C. Deguara, and J. Cooper, Medium-term effects of a monofilament polypropylene mesh for pelvic organ prolapse and sexual function symptoms. Journal of Obstetrics \& Gynaecology, 2012. 32(3): p. 285-290.

36. Celik, D.B., N.K. Beji, and O. Yalcin, Sexual function in women after urinary incontinence and/or pelvic organ prolapse surgery. Journal of Clinical Nursing, 2014. 23(17-18): p. 2637-2648.

37. Chauvin, C., et al., Potential relevance of pre-operative quality of life questionnaires to identify candidates for surgical treatment of genital prolapse: a pilot study. BMC Urol, 2012. 12: p. 9.

38. Chen, Y.S., et al., Midterm prospective comparison of vaginal repair with mesh vs Prolift system devices for prolapse. Eur J Obstet Gynecol Reprod Biol, 2012. 164(2): p. 221-6.

39. Darai, E., et al., Genital prolapse repair using porcine skin implant and bilateral sacrospinous fixation: midterm functional outcome and quality-of-life assessment. Urology, 2009. 73(2): p. 245-50.

40. Demirci, F., et al., Abdominal sacrohysteropexy in young women with uterovaginal prolapse. Journal of Reproductive Medicine, 2006. 51(7): p. 539543.

41. El Haddad, R., et al., Women's quality of life and sexual function after transvaginal anterior repair with mesh insertion. European Journal of Obstetrics \& Gynecology \& Reproductive Biology, 2013. 167(1): p. 110-113.

42. Glavind, K., T. Larsen, and A.S.I. Lindquist, Sexual function in women before and after surgery for pelvic organ prolapse. Acta Obstetricia et Gynecologica Scandinavica, 2015. 94(1): p. 80-85.

43. Hefni, M., et al., Long-term quality of life and patient satisfaction following anterior vaginal mesh repair for cystocele. Archives of Gynecology \& Obstetrics, 2013. 287(3): p. 441-446.

44. Kim, M.R., J.H. Kim, and H.H. Cho, Infracoccygeal sacropexy improves the quality of life of women with uterine prolapse. Maturitas, 2008. 59(2): p. 158-162.

45. Mamik, M.M., et al., Goal attainment after treatment in patients with symptomatic pelvic organ prolapse. American Journal of Obstetrics \& Gynecology, 2013. 209(5): p. 488.e1-5.

46. Murphy, M., et al., Quality of life and surgical satisfaction after vaginal reconstructive vs obliterative surgery for the treatment of advanced pelvic organ prolapse. American Journal of Obstetrics \& Gynecology, 2008. 198(5): p. 573.e17.

47. Perez, T., et al., Laparoscopic sacrocolpopexy for management of pelvic organ prolapse enhances quality of life at one year: a prospective observational study. Journal of Minimally Invasive Gynecology, 2011. 18(6): p. 747-754. 
48. Polin, M.R., et al., Effects of transvaginal repair of symptomatic rectocele on symptom-specific distress and impact on quality of life. International Journal of Gynecology \& Obstetrics, 2012. 117(3): p. 224-227.

49. Sokol, A.I., et al., One-year objective and functional outcomes of a randomized clinical trial of vaginal mesh for prolapse. American Journal of Obstetrics \& Gynecology, 2012. 206(1): p. 86.e1-9.

50. Souviat, C., et al., Long-term functional stability of sacrospinous ligamentfixation repair of pelvic organ prolapse. Journal of Obstetrics \& Gynaecology, 2012. 32(8): p. 781-785.

51. Su, T.-H., et al., Short term impact on female sexual function of pelvic floor reconstruction with the prolift procedure. Journal of Sexual Medicine, 2009. 6(11): p. 3201-3207.

52. van Iersel, J.J., et al., Robot-Assisted Sacrocolporectopexy for Multicompartment Prolapse of the Pelvic Floor: A Prospective Cohort Study Evaluating Functional and Sexual Outcome. Dis Colon Rectum, 2016. 59(10): p. 968-74.

53. van Raalte, H.M., et al., One-year anatomic and quality-of-life outcomes after the Prolift procedure for treatment of posthysterectomy prolapse. Am J Obstet Gynecol, 2008. 199(6): p. 694.e1-6.

54. Vitale, S.G., et al., Biocompatible porcine dermis graft to treat severe cystocele: impact on quality of life and sexuality. Arch Gynecol Obstet, 2016. 293(1): p. 125-31.

55. Yesil, A., D. Watermann, and J. Farthmann, Mesh implantation for pelvic organ prolapse improves quality of life. Arch Gynecol Obstet, 2014. 289(4): p. 817-21.

56. Buca, D.I., et al., A 2-Year Evaluation of Quality of Life Outcomes of Patients With Pelvic Organ Prolapse Treated With an Elevate Prolapse Repair System. Female Pelvic Med Reconstr Surg, 2016. 22(6): p. 410-414.

57. Farthmann, J., et al., Improvement of pelvic floor-related quality of life and sexual function after vaginal mesh implantation for cystocele: primary endpoint of a prospective multicentre trial. Archives of Gynecology \& Obstetrics, 2016. 294(1): p. 115-121.

58. Funfgeld, C., et al., Quality of Life, Sexuality, Anatomical Results and Side-effects of Implantation of an Alloplastic Mesh for Cystocele Correction at Follow-up after 36 Months. Geburtshilfe Frauenheilkd, 2017. 77(9): p. 993-1001.

59. Grimminck, K., et al., Long-term follow-up and quality of life after robot assisted sacrohysteropexy. European Journal of Obstetrics \& Gynecology \& Reproductive Biology, 2016. 206: p. 27-31.

60. Laas, E., et al., Preoperative quality of life questionnaires are an adequate tool to select women with genital prolapse for laparoscopic sacrocolpopexy. Int Urogynecol J, 2017.

61. Lukacz, E.S., et al., Quality of Life and Sexual Function 2 Years After Vaginal Surgery for Prolapse. Obstetrics \& Gynecology, 2016. 127(6): p. 1071-1079.

62. Rahkola-Soisalo, P., et al., Quality of life after Uphold ${ }^{\mathrm{TM}}$ Vaginal Support System surgery for apical pelvic organ prolapse-A prospective multicenter study.

European Journal of Obstetrics \& Gynecology \& Reproductive Biology, 2017. 208: p. 86-90. 
63. Schiavi, M.C., et al., Vaginal Native Tissue Repair for Posterior Compartment Prolapse: Long-Term Analysis of Sexual Function and Quality of Life in 151 Patients. Female Pelvic Med Reconstr Surg, 2017.

64. Vallabh-Patel, V., C. Saiz, and C. Salamon, Subjective and Objective Outcomes of Robotic and Vaginal High Uterosacral Ligament Suspension. Female Pelvic Med Reconstr Surg, 2016. 22(6): p. 420-424.

65. Gupta, P., et al., Analysis of changes in sexual function in women undergoing pelvic organ prolapse repair with abdominal or vaginal approaches. Int Urogynecol J, 2016. 27(12): p. 1919-1924.

66. Richter, H.E., et al., The effect of age on short-term outcomes after abdominal surgery for pelvic organ prolapse. J Am Geriatr Soc, 2007. 55(6): p. 857-63.

67. Asoğlu, M.R., et al., Colpocleisis, patient satisfaction and quality of life. Journal of the Turkish-German Gynecological Association, 2012. 13(4): p. 253-256.

68. Hullfish, K.L., V.E. Bovbjerg, and W.D. Steers, Colpocleisis for pelvic organ prolapse: patient goals, quality of life, and satisfaction. Obstet Gynecol, 2007. 110(2 Pt 1): p. 341-5.

69. Yeniel, A.O., et al., Quality of life scores improve in women undergoing colpocleisis: a pilot study. Eur J Obstet Gynecol Reprod Biol, 2012. 163(2): p. 230-3.

70. Wang, X., Y. Chen, and K. Hua, Pelvic Symptoms, Body Image, and Regret after LeFort Colpocleisis: A Long-Term Follow-Up. Journal of Minimally Invasive Gynecology, 2017. 24(3): p. 415-419.

71. Cundiff, G.W., et al., The PESSRI study: symptom relief outcomes of a randomized crossover trial of the ring and Gellhorn pessaries. Am J Obstet Gynecol, 2007. 196(4): p. 405.e1-8.

72. Ko, P.C., et al., Use of a pessary in treatment of pelvic organ prolapse: quality of life, compliance, and failure at 1-year follow-up. J Minim Invasive Gynecol, 2011. 18(1): p. 68-74.

73. Patel, M., et al., Impact of pessary use on prolapse symptoms, quality of life, and body image. Am J Obstet Gynecol, 2010. 202(5): p. 499.e1-4.

74. Ahadi, T., et al., Efficacy of biofeedback on quality of life in stages I and II pelvic organ prolapse: A Pilot study. Eur J Obstet Gynecol Reprod Biol, 2017. 215: p. 241-246.

75. Bartuzi, A., et al., Self-perceived quality of life after pelvic organ prolapse reconstructive mesh surgery: prospective study. Eur J Obstet Gynecol Reprod Biol, 2013. 169(1): p. 108-12.

76. de Oliveira, M.S., A. Cavalcanti Gde, and A.A. da Costa, Fascial surgical repair for vaginal prolapse: effect on quality of life and related symptoms. Eur J Obstet Gynecol Reprod Biol, 2014. 182: p. 177-80.

77. Dhital, R., et al., Improved quality of life after surgery for pelvic organ prolapse in Nepalese women. BMC Womens Health, 2013. 13: p. 22.

78. Lowenstein, L., et al., Changes in sexual function after treatment for prolapse are related to the improvement in body image perception. J Sex Med, 2010. 7(2 Pt 2): p. 1023-8. 
79. Caruso, S., et al., Effects of ultralow topical estriol dose on vaginal health and quality of life in postmenopausal women who underwent surgical treatment for pelvic organ prolapse. Menopause, 2017. 24(8): p. 900-907.

80. Crisp, C.C., et al., Body image, regret, and satisfaction following colpocleisis. Am J Obstet Gynecol, 2013. 209(5): p. 473.e1-7.

81. Pakbaz, M., I. Mogren, and M. Lofgren, Outcomes of vaginal hysterectomy for uterovaginal prolapse: a population-based, retrospective, cross-sectional study of patient perceptions of results including sexual activity, urinary symptoms, and provided care. BMC Womens Health, 2009. 9: p. 9.

82. Sun, Z., et al., Medium-term outcomes after combined trachelectomy and uterosacral ligament suspension among young women with severe uterine prolapse. Int J Gynaecol Obstet, 2016. 132(2): p. 224-8.

83. Veit-Rubin, N., et al., Patient satisfaction after laparoscopic lateral suspension with mesh for pelvic organ prolapse: outcome report of a continuous series of 417 patients. Int Urogynecol J, 2017. 28(11): p. 1685-1693.

84. Kenton, K., et al., Patient preparedness: an important predictor of surgical outcome. Am J Obstet Gynecol, 2007. 197(6): p. 654.e1-6.

85. Srikrishna, S., D. Robinson, and L. Cardozo, A longitudinal study of patient and surgeon goal achievement 2 years after surgery following pelvic floor dysfunction surgery. Bjog, 2010. 117(12): p. 1504-11.

86. Vollebregt, A., et al., Effects of vaginal prolapse surgery on sexuality in women and men; Results from a RCT on repair with and without mesh. Journal of Sexual Medicine, 2012. 9(4): p. 1200-1211.

87. Azar, M., et al., Sexual function in women after surgery for pelvic organ prolapse. International Urogynecology Journal, 2008. 19(1): p. 53-57.

88. Brandner, S., et al., Sexual function after rectocele repair. Journal of Sexual Medicine, 2011. 8(2): p. 583-588.

89. Kuhn, A., et al., Male and female sexual function after surgical repair of female organ prolapse. Journal of Sexual Medicine, 2009. 6(5): p. 1324-1334.

90. Kuhn, A., et al., Sexual function after sacrocolpopexy. Journal of Sexual Medicine, 2010. 7(12): p. 4018-4023.

91. Detollenaere, R.J., et al., The impact of sacrospinous hysteropexy and vaginal hysterectomy with suspension of the uterosacral ligaments on sexual function in women with uterine prolapse: A secondary analysis of a randomized comparative study. Journal of Sexual Medicine, 2016. 13(2): p. 213-219.

92. Uçar, M.G., et al., Sexual functioning before and after vaginal hysterectomy to treat pelvic organ prolapse and the effects of vaginal cuff closure techniques: a prospective randomised study. European Journal of Obstetrics \& Gynecology \& Reproductive Biology, 2016. 206: p. 1-5.

93. Roovers, J.-P., et al., Effects of genital prolapse surgery on sexuality. Journal of Psychosomatic Obstetrics \& Gynecology, 2006. 27(1): p. 43-48.

94. Bartuzi, A., et al., Transvaginal Prolift((R)) mesh surgery due to advanced pelvic organ prolapse does not impair female sexual function: a prospective study. European Journal of Obstetrics \& Gynecology \& Reproductive Biology, 2012. 165(2): p. 295-298. 
95. Jeng, C., et al., Sexual functioning after vaginal hysterectomy or transvaginal sacrospinous uterine suspension for uterine prolapse: a comparison. Journal of Reproductive Medicine, 2005. 50(9): p. 669-674.

96. Lonnée-Hoffmann, R.A.M., et al., What predicts improvement of sexual function after pelvic floor surgery? A follow-up study. Acta Obstetricia et Gynecologica Scandinavica, 2013. 92(11): p. 1304-1312.

97. Milani, A.L., et al., Sexual function following trocar-guided mesh or vaginal native tissue repair in recurrent prolapse: A randomized controlled trial. Journal of Sexual Medicine, 2011. 8(10): p. 2944-2953.

98. Zucchi, A., et al., Female sexual dysfunction in urogenital prolapse surgery: Colposacropexy vs. hysterocolposacropexy. Journal of Sexual Medicine, 2008. 5(1): p. 139-145.

99. Ko, Y.C., et al., Comparison of sexual function between sacrocolpopexy and sacrocervicopexy. Obstet Gynecol Sci, 2017. 60(2): p. 207-212.

100. Long, C.-Y., et al., Changes in female sexual function following anterior with and without posterior vaginal mesh surgery for the treatment of pelvic organ prolapse. Journal of Sexual Medicine, 2012. 9(8): p. 2167-2174.

101. Long, C.Y., et al., Comparison of the changes in sexual function of premenopausal and postmenopausal women following transvaginal mesh surgery. Journal of Sexual Medicine, 2011. 8(7): p. 2009-2016.

102. Brækken, I.H., et al., Can pelvic floor muscle training improve sexual function in women with pelvic organ prolapse? A randomized controlled trial. Journal of Sexual Medicine, 2015. 12(2): p. 470-480.

103. Kuhn, A., et al., Sexual and organ function in patients with symptomatic prolapse: are pessaries helpful? Fertil Steril, 2009. 91(5): p. 1914-8.

104. Meriwether, K.V., et al., Sexual Function and Pessary Management among Women Using a Pessary for Pelvic Floor Disorders. J Sex Med, 2015. 12(12): p. 2339-49.

105. Hoda, M.R., et al., Prospective follow-up of female sexual function after vaginal surgery for pelvic organ prolapse using transobturator mesh implants. J Sex Med, 2011. 8(3): p. 914-22.

106. Dua, A., et al., The effect of prolapse repair on sexual function in women. Journal of Sexual Medicine, 2012. 9(5): p. 1459-1465.

107. Altman, D., et al., Sexual dysfunction after trocar-guided transvaginal mesh repair of pelvic organ prolapse. Obstet Gynecol, 2009. 113(1): p. 127-33.

108. Ghetti, C., et al., Depressive symptoms in women seeking surgery for pelvic organ prolapse. Int Urogynecol J, 2010. 21(7): p. 855-60.

109. Liebergall-Wischnitzer, M., et al., Women's well-being after Manchester procedure for pelvic reconstruction with uterine preservation: a follow-up study. Arch Gynecol Obstet, 2012. 285(6): p. 1587-92.

110. Klapdor, R., et al., Postoperative anatomic and quality-of-life outcomes after vaginal sacrocolporectopexy for vaginal vault prolapse. International Journal of Gynecology \& Obstetrics, 2017. 137(1): p. 86-91.

111. O'Dell, K.K. and C.S. Jacelon, Not the surgery for a young person: women's experience with vaginal closure surgery for severe prolapse. Urol Nurs, 2005. 25(5): p. 345-51. 
112. Katsara, A., et al., Long-term quality of life, satisfaction, pelvic floor symptoms and regret after colpocleisis. Arch Gynecol Obstet, 2016. 294(5): p. 999-1003.

113. Wheeler, T.L., 2nd, et al., Regret, satisfaction, and symptom improvement: analysis of the impact of partial colpocleisis for the management of severe pelvic organ prolapse. Am J Obstet Gynecol, 2005. 193(6): p. 2067-70.

114. Larson, K.A., et al., Long-term patient satisfaction with michigan four-wall sacrospinous ligament suspension for prolapse. Obstet Gynecol, 2013. 122(5): p. 967-75.

115. Basu, M. and J.R. Duckett, Barriers to seeking treatment for women with persistent or recurrent symptoms in urogynaecology. BJOG: An International Journal of Obstetrics \& Gynaecology, 2009. 116(5): p. 726-730.

116. Fritel, X., et al., Symptomatic pelvic organ prolapse at midlife, quality of life, and risk factors. Obstetrics \& Gynecology, 2009. 113(3): p. 609-616.

117. Kinman, C.L., et al., The relationship between age and pelvic organ prolapse bother. Int Urogynecol J, 2017. 28(5): p. 751-755.

118. Novi, J.M., et al., Sexual function in women with pelvic organ prolapse compared to women without pelvic organ prolapse. J Urol, 2005. 173(5): p. 1669-72.

119. Lowenstein, L., et al., Sexual function is related to body image perception in women with pelvic organ prolapse. J Sex Med, 2009. 6(8): p. 2286-91.

120. Zielinski, R., et al., The relationship between pelvic organ prolapse, genital body image, and sexual health. Neurourol Urodyn, 2012. 31(7): p. 1145-8.

121. Ai, F., et al., Depressive symptoms screening in postmenopausal women with symptomatic pelvic organ prolapse. Menopause, 2017.

122. Fabre, L.F. and L.C. Smith, The effect of major depression on sexual function in women. Journal of Sexual Medicine, 2012. 9(1): p. 231-239.

123. Mondloch, M.V., D.C. Cole, and J.W. Frank, Does how you do depend on how you think you'll do? A systematic review of the evidence for a relation between patients' recovery expectations and health outcomes. Cmaj, 2001. 165(2): p. 1749.

124. Yaakobi, T., et al., Direct and Indirect Effects of Personality Traits on Psychological Distress in Women With Pelvic Floor Disorders. Female Pelvic Med Reconstr Surg, 2017. 23(6): p. 412-416.

125. Pakarinen, M., et al., Life dissatisfaction is associated with depression and poorer surgical outcomes among lumbar spinal stenosis patients: a 10-year follow-up study. Int J Rehabil Res, 2016. 39(4): p. 291-295.

126. Waldrop, D., et al., Self-efficacy, optimism, health competence, and recovery from orthopedic surgery. Journal of Counseling Psychology, 2001. 48(2): p. 233-238.

127. Ronaldson, A., et al., Optimism measured pre-operatively is associated with reduced pain intensity and physical symptom reporting after coronary artery bypass graft surgery. J Psychosom Res, 2014. 77(4): p. 278-82.

128. Mendelson, B.K., M.J. Mendelson, and D.R. White, Body-esteem scale for adolescents and adults. J Pers Assess, 2001. 76(1): p. 90-106.

129. Hopwood, P., et al., A body image scale for use with cancer patients. Eur J Cancer, 2001. 37(2): p. 189-97.

130. Zielinski, R.E., et al., Validity and reliability of a scale to measure genital body image. J Sex Marital Ther, 2012. 38(4): p. 309-24. 
131. EuroQol--a new facility for the measurement of health-related quality of life. Health Policy, 1990. 16(3): p. 199-208.

132. Hunt, S.M., J. McEwen, and S.P. McKenna, Measuring health status: a new tool for clinicians and epidemiologists. J R Coll Gen Pract, 1985. 35(273): p. 185-8.

133. Ware, J., Jr., M. Kosinski, and S.D. Keller, A 12-Item Short-Form Health Survey: construction of scales and preliminary tests of reliability and validity. Med Care, 1996. 34(3): p. 220-33.

134. Murphy, B., et al., Australian WHOQoL instruments: User's manual and interpretation guide. 2000.

135. Parker, R.M., et al., The test of functional health literacy in adults: a new instrument for measuring patients' literacy skills. J Gen Intern Med, 1995. 10(10): p. 537-41.

136. Jackson, S., et al., The Bristol Female Lower Urinary Tract Symptoms questionnaire: development and psychometric testing. Br J Urol, 1996. 77(6): p. 805-12.

137. Baessler, K. and C. Kempkensteffen, [Validation of a comprehensive pelvic floor questionnaire for the hospital, private practice and research]. Gynakol Geburtshilfliche Rundsch, 2009. 49(4): p. 299-307.

138. Avery, K., et al., ICIQ: a brief and robust measure for evaluating the symptoms and impact of urinary incontinence. Neurourol Urodyn, 2004. 23(4): p. 322-30.

139. Price, N., et al., Development and psychometric evaluation of the ICIQ Vaginal Symptoms Questionnaire: the ICIQ-VS. Bjog, 2006. 113(6): p. 700-12.

140. Shumaker, S.A., et al., Health-related quality of life measures for women with urinary incontinence: the Incontinence Impact Questionnaire and the Urogenital Distress Inventory. Continence Program in Women (CPW) Research Group. Qual Life Res, 1994. 3(5): p. 291-306.

141. Uebersax, J.S., et al., Short forms to assess life quality and symptom distress for urinary incontinence in women: the Incontinence Impact Questionnaire and the Urogenital Distress Inventory. Continence Program for Women Research Group. Neurourol Urodyn, 1995. 14(2): p. 131-9.

142. Sandvik, H., et al., Validation of a severity index in female urinary incontinence and its implementation in an epidemiological survey. J Epidemiol Community Health, 1993. 47(6): p. 497-9.

143. Nelson, C.P., et al., Incontinence Symptom Index-Pediatric: development and initial validation of a urinary incontinence instrument for the older pediatric population. J Urol, 2007. 178(4 Pt 2): p. 1763-7; discussion 1767.

144. Acquadro, C., et al., Translating overactive bladder questionnaires in 14 languages. Urology, 2006. 67(3): p. 536-40.

145. Pescatori, M., et al., New grading and scoring for anal incontinence. Evaluation of 335 patients. Dis Colon Rectum, 1992. 35(5): p. 482-7.

146. Barber, M.D., et al., Psychometric evaluation of 2 comprehensive conditionspecific quality of life instruments for women with pelvic floor disorders. Am J Obstet Gynecol, 2001. 185(6): p. 1388-95.

147. Yalcin, I. and R.C. Bump, Validation of two global impression questionnaires for incontinence. Am J Obstet Gynecol, 2003. 189(1): p. 98-101. 
148. Bradshaw, H.D., et al., Development and psychometric testing of a symptom index for pelvic organ prolapse. J Obstet Gynaecol, 2006. 26(3): p. 241-52.

149. Bradley, C.S., et al., The questionnaire for urinary incontinence diagnosis (QUID): validity and responsiveness to change in women undergoing nonsurgical therapies for treatment of stress predominant urinary incontinence. Neurourol Urodyn, 2010. 29(5): p. 727-34.

150. Beck, A.T., et al., Comparison of Beck Depression Inventories -IA and -II in psychiatric outpatients. J Pers Assess, 1996. 67(3): p. 588-97.

151. Goldberg, D., Manual of the general health questionnaire. 1978, Windsor: NFER.

152. Kroenke, K., R.L. Spitzer, and J.B.W. Williams, The PHQ-9: Validity of a brief depression severity measure. Journal of General Internal Medicine, 2001. 16(9): p. 606-613.

153. Sung, V.W., et al., Validation of decision-making outcomes for female pelvic floor disorders. Am J Obstet Gynecol, 2008. 198(5): p. 575.e1-6.

154. O'Leary, M.P., et al., A brief male sexual function inventory for urology. Urology, 1995. 46(5): p. 697-706.

155. Rosen, R., et al., The Female Sexual Function Index (FSFI): a multidimensional self-report instrument for the assessment of female sexual function. J Sex Marital Ther, 2000. 26(2): p. 191-208.

156. Rust, J. and S. Golombok, The GRISS: a psychometric instrument for the assessment of sexual dysfunction. Arch Sex Behav, 1986. 15(2): p. 157-65.

157. Rosen, R.C., et al., Male Sexual Health Questionnaire (MSHQ): scale development and psychometric validation. Urology, 2004. 64(4): p. 777-82.

158. Rogers, R.G., et al., A new instrument to measure sexual function in women with urinary incontinence or pelvic organ prolapse. Am J Obstet Gynecol, 2001. 184(4): p. 552-8.

159. Rogers, R.G., et al., A short form of the Pelvic Organ Prolapse/Urinary Incontinence Sexual Questionnaire (PISQ-12). Int Urogynecol J Pelvic Floor Dysfunct, 2003. 14(3): p. 164-8; discussion 168.

160. Rogers, R.G., et al., A new measure of sexual function in women with pelvic floor disorders (PFD): the Pelvic Organ Prolapse/Incontinence Sexual Questionnaire, IUGA-Revised (PISQ-IR). Int Urogynecol J, 2013. 24(7): p. 1091-103.

161. Vroege, J., Vragenlijst voor het signaleren van Seksuele Dysfuncties (VSD), (5 deversie) [Questionnaire for screening Sexual Dysfunctions (QSD), (5th ed.)], ed. Utrecht. 1994, The Netherlands. 
Figure 1: Article Selection. PRISMA style flow-chart of article inclusion and exclusion through each phase of the screening process. 